\title{
RELATIONSHIP BETWEEN CYTOTOXICITY AND CONVERSION OF THIOSANGIVAMYCIN ANALOGS TO TOYOCAMYCIN ANALOGS IN CELL CULTURE MEDIUM
}

\author{
Thomas E. Renau, James S. Lee, I Ianna Kim, Christopher G. Young, \\ Linda L. WOTRING, LEROY B. TOWNSEND and JOHN C. DRACH* \\ Interdepartmental Graduate Program in Medicinal Chemistry, College of Pharmacy, and \\ Department of Biologic and Materials Sciences, School of Dentistry, University of Michigan, \\ Ann Arbor, MI 48109-1078, U.S.A.
}

(Received 17 January 1994; accepted 30 March 1994)

\begin{abstract}
Non-nucleoside analogs of the pyrrolopyrimidine nucleosides toyocamycin, sangivamycin and thiosangivamycin have been synthesized and their cytotoxicity in mammalian cells determined. While studying the effects of 5-thioamide-substituted analogs on cell growth, we observed an interesting phenomenon in which cells recovered spontaneously from growth inhibition during extended incubations. HPLC studies demonstrated that the 5-thioamide moiety of several structurally dissimilar 7-substituted 4-aminopyrrolo[2,3- $d]$ pyrimidines, including thiosangivamycin, is unstable in cell culture medium and is converted to the corresponding 5-nitrile with a half-life of approximately $48 \mathrm{hr}$. In contrast, different substituents at the 4-position of the heterocycle significantly affected the stability of the 5-thioamide moiety. Conversion of the thioamide to the nitrile was caused by components in the cell culture medium, not components of serum. The above observations demonstrate that caution should be exercised in interpreting biological data obtained in vitro for 5-thioamide pyrrolo[2,3-d]pyrimidines.
\end{abstract}

Key words: pyrrolopyrimidine; non-nucleoside; cytotoxicity; stability; HPLC

The pyrrolo[2,3- $d]$ pyrimidine nucleoside antibiotics toyocamycin (1a, Table 1) and sangivamycin (1b) originally were isolated from different strains of streptomyces in 1956 and 1963 , respectively $[1,2]$. The total synthesis of these compounds was reported in 1969 by Townsend and coworkers [3]. A synthetic analog, thiosangivamycin (1c), was described in 1970 by this same research group [4]. All three compounds $(1 \mathrm{a}-\mathrm{c})$ are highly toxic to mammalian cells and have been evaluated as potential anti-cancer agents $[5,6]$. Sangivamycin was advanced to phase I clinical trials, and a report on its toxicity has been published [7].

As part of our ongoing research involving pyrrolo $[2,3-d]$ pyrimidines as potential antiviral agents [8-14], we recently described the synthesis and activity against $\mathrm{HCMV} \dagger$ of a number of nonnucleoside derivatives related to toyocamycin, sangivamycin and thiosangivamycin $(1 \mathrm{a}-\mathrm{c})$ [15]. We have expanded our studies in this area and now have synthesized a number of closely related compounds (2-6). While studying the cytotoxicity profile of these new analogs, we observed an unusual phenomenon in which cells spontaneously recovered from growth inhibition during extended incubation times. An

* Corresponding author: Dr. J. C. Drach, School of Dentistry, University of Michigan, Ann Arbor, MI 48109-1078. Tel. (313) 763-5481; FAX (313) 764-7406.

† Abbreviations: HCMV, human cytomegalovirus; MEM, minimal essential medium; PDT, population doubling time; and HBS, HEPES-buffered salt solution. investigation and an explanation of this phenomenon follows.

\section{MATERIALS AND METHODS}

Materials. Toyocamycin, sangivamycin and thiosangivamycin were obtained from the library of compounds in the laboratory of one of us (L.B.T.). Compounds 2 and 3 were synthesized in four or five steps from the known 2-amino-5-bromo-3,4dicyanopyrrole [16] by a modification of the procedure described in Ref. 15. Compounds 4-6 were synthesized from the key intermediate 4-chloro7 - [(2 - methoxyethoxy)methyl $]$ pyrrolo $[2,3-d]$ pyrimidine-5-carbonitrile generated by a modification of the procedure described previously [17]. No impurities were detected in the ${ }^{1} \mathrm{H}$ NMR of all compounds examined and C-H-N analysis of these compounds was within the acceptable $\pm 0.4 \%$ of theoretical values. Details of the synthetic procedures, physical characteristics, and antiviral activity of compounds 2-6 are being published elsewhere.

Cell cultures. KB cells, an established human cell linc derived from an epidermal oral carcinoma, were grown in MEM supplemented with $10 \%$ calf serum and were subcultured by conventional methods by using $0.05 \%$ trypsin plus $0.02 \%$ EDTA [8]. The average PDT for $\mathrm{KB}$ cells in the absence of drugs was $21 \mathrm{hr}$. L1210 murine leukemic cells were grown in Fischer's medium supplemented with $10 \%$ heat- 
Table 1. Structure and cytotoxicity of 4-aminopyrrolo[2,3- $d]$ pyrimidine analogs

\begin{tabular}{|c|c|c|c|c|}
\hline & & $\int^{R_{1}}$ & & \\
\hline \multirow{2}{*}{ Compound } & \multirow[b]{2}{*}{$\mathbf{R}_{1}$} & \multirow[b]{2}{*}{$\mathbf{R}_{2}$} & \multicolumn{2}{|c|}{ Cytotoxicity, $\mathrm{IC}_{50}(\mu \mathrm{M})$} \\
\hline & & & $\mathrm{KB}$ cells & L1210 cells \\
\hline $1 \mathbf{a}$ & $\mathrm{CN}$ & $\beta$-D-Ribofuranose & 0.03 & 0.004 \\
\hline b & $\mathrm{CONH}_{2}$ & $\beta$-D-Ribofuranose & 0.06 & 0.003 \\
\hline c & $\mathrm{CSNH}_{2}$ & $\beta$-D-Ribofuranose & 0.08 & 0.023 \\
\hline $2 \mathbf{a}$ & $\mathrm{CN}$ & $\mathrm{CH}_{2} \mathrm{C}_{6} \mathrm{H}_{5}$ & $>100$ & $>100$ \\
\hline b & $\mathrm{CONH}_{2}$ & $\mathrm{CH}_{2} \mathrm{C}_{6} \mathrm{H}_{5}$ & 84 & 16 \\
\hline c & $\mathrm{CSNH}_{2}$ & $\mathrm{CH}_{2} \mathrm{C}_{6} \mathrm{H}_{5}$ & 18 & 70 \\
\hline $\mathbf{3 a}$ & $\mathrm{CN}$ & $\mathrm{CH}_{2} \mathrm{O}\left(\mathrm{CH}_{2}\right)_{2} \mathrm{OCH}_{3}$ & $>100$ & $>100$ \\
\hline b & $\mathrm{CONH}_{2}$ & $\mathrm{CH}_{2} \mathrm{O}\left(\mathrm{CH}_{2}\right)_{2} \mathrm{OCH}_{3}$ & $>100$ & $>100$ \\
\hline c & $\mathrm{CSNH}_{2}$ & $\mathrm{CH}_{2} \mathrm{O}\left(\mathrm{CH}_{2}\right)_{2} \mathrm{OCH}_{3}$ & $>100$ & $>100$ \\
\hline
\end{tabular}

Cytotoxicity was determined by measuring the effects of compounds on the growth of KB and L1210 cells. Data are the averages of two or more experiments. The greater than symbol indicates that the $\mathrm{IC}_{50}$ was not reached at the highest concentration tested. Compounds la-c are toyocamycin, sangivamycin and thiosangivamycin, respectively.

inactivated $\left(56^{\circ}, 30 \mathrm{~min}\right)$ horse serum and were subcultured by serial dilution. All cell lines were screened periodically for Mycoplasma contamination and were negative.

Cytotoxicity studies. The cytotoxicity of compounds $\mathbf{1 a - c}$ to $3 \mathbf{a}-\mathbf{c}$ was determined initially in $\mathrm{KB}$ cells by a staining method previously described [18] and in an L1210 cell growth assay [19]. In more detailed studies, the inhibitory effect of compound $2 c$ was evaluated and PDTs were determined in a $\mathrm{KB}$ cell growth assay [20]. PDTs are significantly longer in cells whose growth is inhibited, or whose absolute numbers decrease as a result of toxicity affecting their growth. PDTs were calculated by means of a least squares program fitting the exponential portion of a growth curve. For growth studies with KB and L1210 cells, growth rates were calculated from determinations of the number of cells at $0,24,48$, 72 and $96 \mathrm{hr}$ in the presence of selected concentrations of the test compound. Cells were enumerated using a Coulter Counter (Coulter Electronics, Hialeah, FL). Growth inhibition was calculated as the slope of a semi-logarithmic plot of cell number against time for the treated culture as a percent of the control. In both the staining and cell growth studies, the $\mathrm{IC}_{50}$ was defined as the concentration required to decrease the growth rate to $50 \%$ of the control rate.

Compound stability assays. The stability of compounds in cell culture medium was investigated by incubating $100 \mu \mathrm{M}$ concentrations of compounds $1-6$ at $37^{\circ}$ in tightly capped tubes. The compounds were incubated in either Fischer's medium, MEM, or HBS [21] (10 mM HEPES, $137 \mathrm{mM} \mathrm{NaCl}, 5.4 \mathrm{mM}$ $\mathrm{KCl}, 0.7 \mathrm{mM} \mathrm{Na} \mathrm{HPO}_{4}, 5.6 \mathrm{mM}$ dextrose, $\mathrm{pH} 6.5$ ). In all cases, the incubation media contained neither
Table 2. Retention times and UV absorbance data of pyrrolo[2,3-d]pyrimidine analogs

\begin{tabular}{lcc}
\hline Compound & RT (min) & $\lambda_{\max }(\mathbf{n m})$ \\
\hline $\mathbf{1 a}$ & 12 & 274 \\
$\mathbf{b}$ & 10.5 & 278 \\
c & 11 & 292 \\
$\mathbf{2 a}$ & 45 & 277 \\
$\mathbf{b}$ & 46 & 278 \\
c & 50 & 294 \\
$\mathbf{3 a}$ & 22 & 273 \\
b & 25 & 275 \\
c & 28 & 292 \\
$\mathbf{4 a}$ & 27 & 281 \\
b & 33 & 286 \\
$\mathbf{5 a}$ & 33 & 290 \\
b & 26 & 289 \\
$\mathbf{6 a}$ & 28 & 271 \\
b & 24 & 266 \\
\hline
\end{tabular}

Retention times (RT) were determined by reverse-phase HPLC as described in the text. The $\lambda_{\max }(\mathrm{nm})$ of each compound was determined by UV spectroscopy.

cells nor serum. The $\mathrm{pH}$ of the medium was monitored and was found to be consistently between 7.5 and 7.8. At selected times during periods extending up to approximately 3 weeks, $100-\mu \mathrm{L}$ aliquots of medium or buffer were removed and immediately analyzed by reverse-phase HPLC.

Analytical procedures. HPLC of compounds 1-6 was performed with a Beckman Ultrasphere ODS reverse-phase column $(5 \mu \mathrm{m}, 4.6 \mathrm{~mm} \times 15 \mathrm{~cm})$. HPLC analysis of la-c was adapted from a literature 
procedure [22]. Compounds 1a-c were eluted in 30 min using a linear gradient starting with $0.02 \mathrm{M}$ $\mathrm{KH}_{2} \mathrm{PO}_{4}$ (pH 5.6): $60 \% \mathrm{MeOH} / 40 \% \mathrm{H}_{2} \mathrm{O}$ solution $(87: 13, v / v)$ and ending with $100 \%$ of the $60 \%$ $\mathrm{MeOH} / 40 \% \mathrm{H}_{2} \mathrm{O}$ solution. Elution of compounds 2-6 was accomplished in $70 \mathrm{~min}$ using a linear gradient starting with $0.1 \%$ trifluoroacetic acid in $\mathrm{H}_{2} \mathrm{O}$ and ending with $50 \% \mathrm{MeOH}$. UV absorbance of the compounds was monitored simultaneously at the $\lambda_{\max }$ for the thioamide and nitrile substituted derivatives (Table 2). Retention times (RT) for compounds $1-6$ are shown in Table 2. Peak areas were determined using System Gold Software (Beckman Instruments, Fullerton, CA). The purity of each compound was determined by HPLC before the start of each experiment.

\section{RESULTS}

7-Benzyl-substituted 4-aminopyrrolo[2,3-d]pyrimidine derivatives $(\mathbf{2 a - c}$, Table 1$)$, related to toyocamycin (1a), sangivamycin (1b) and thiosangivamycin (1c), were synthesized and examined for activity against HCMV as potential antiviral agents. Results, described elsewhere [23], showed that similar to our previous report on 7-alkyl 4-aminopyrrolo[2,3-d]pyrimidines [15], only the thioamide-substituted derivative $(\mathbf{2 c})$ exhibited activity against HCMV [23]. We have expanded the cytotoxicity portion of those studies and now report that the thioamide $(\mathbf{2 c})$ was more toxic than the carboxamide ( 2 b) in KB cells, but in L1210 cells $\mathbf{2 b}$ was more toxic than $2 \mathrm{c}$ (Table 1 ). In both cell lines, the nitrile derivative (2a) was the least toxic. Nonetheless, compounds $2 a-c$ were substantially less toxic in both KB and L1210 cells when compared with the parent nucleoside analogs $\mathbf{1 a}-\mathbf{c}$. Compounds 3a-c containing a 2-methoxyethoxy methyl side chain at $\mathrm{N}-7$, were the least toxic compounds of those studied.

More detailed studies on the effect of compound $2 \mathrm{c}$ on the growth of uninfected KB cells demonstrated that it suppressed growth at 10 and $32 \mu \mathrm{M}$ (Fig. 1). Inhibition observed at $32 \mu \mathrm{M}$ was reversible upon removal of the drug following a 24-hr incubation period (Fig. 1), suggesting that it acts as a cytostatic rather than a cytotoxic agent. Surprisingly, growth resumed even in cultures from which drug was not removed (Fig. 1). This observation was supported by data from a separate experiment in $\mathrm{KB}$ cells where PDTs were calculated at 24-hr intervals up to $72 \mathrm{hr}$ after incubation of $32 \mu \mathrm{M} \mathrm{2c}$. Over this $72-\mathrm{hr}$ period, the PDT increased from $21 \mathrm{hr}$ in the control to $56 \mathrm{hr}$ in drug-treated cultures during the first 24 hr incubation with $2 c$. In contrast, the PDT of the drug-treated cultures returned to control values ( $20 \mathrm{hr}$ compared with $21 \mathrm{hr}$ in the control) during the last $24-\mathrm{hr}$ interval. Similar results were obtained in L1210 cells. Together these results suggested that $\mathbf{2 c}$ was being converted to a less toxic compound in vitro. Various possibilities were considered for the basis for this recovery: (i) inactivation of the compound by cellular enzymes, (ii) inactivation by enzymes in the serum, or (iii) degradation in cell culture medium. Cell growth recovery could not be totally attributed to cellular or serum proteins

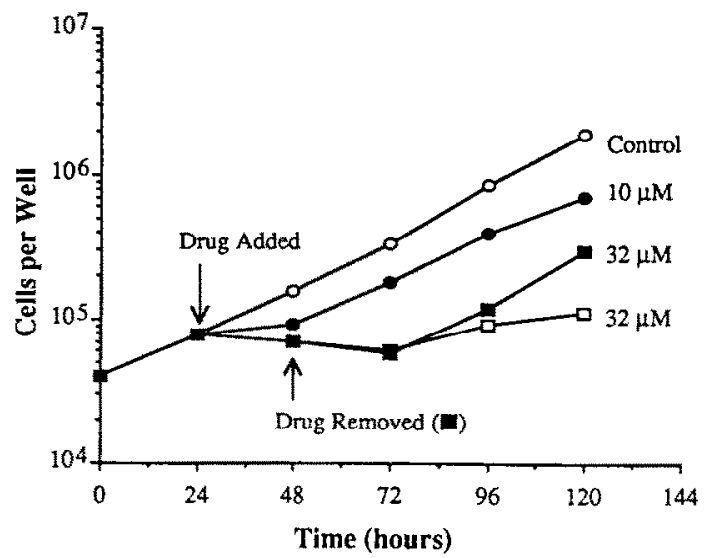

Fig. 1. Effect of compound $2 \mathrm{c}$ on the growth of $\mathrm{KB}$ cells. Cells were seeded at 40,000 cells/well and incubated for $24 \mathrm{hr}$ at which time 10 or $32 \mu \mathrm{M} 2 \mathrm{c}$ was added to three sets of cultures $(\mathbf{Q}, \square, \square)$ and incubated for an additional $24 \mathrm{hr}$. At that time, the medium was removed from one set of cultures containing $32 \mu \mathrm{M}$ drug ( $\mathbf{C}$ ), rinsed with HBS, and the incubation continued with drug-free medium. Cells were harvested at the time periods indicated and enumerated with a Coulter Counter.

inactivating the compound since preincubation of $100 \mu \mathrm{M}$ compound $2 \mathrm{c}$ in medium without serum for 2 days before the addition of cells eliminated inhibition of L1210 cell growth. In this experiment, L1210 cell growth in drug-treated cultures was comparable to the control values even though the concentration of $2 \mathrm{c}$ used was approximately 1.5 times the $\mathrm{IC}_{50}$. Therefore, we concluded that compound $2 c$ was unstable in cell culture medium alone (without serum) and was spontaneously converting to a relatively non-toxic compound.

To investigate this phenomenon, $100 \mu \mathrm{M}$ compound $2 \mathrm{c}$ was incubated in serum-free Fischer's cell culture medium. HPLC analysis was performed on samples of cell culture medium removed at 24-hr intervals. Within the first $48 \mathrm{hr}$ of incubation, more than half of $2 \mathrm{c}$ was converted to a new product (Fig. 2). By $144 \mathrm{hr}$, nearly all of $2 \mathrm{c}$ was converted to this new product. HPLC analysis using both $\mathbf{2 a}$ and $\mathbf{2 b}$ as markers showed that this new peak co-eluted with the corresponding nitrile (2a). From this it was calculated that the thioamide (2c) was converted to $2 a$ with a $T_{1 / 2}$ of $41 \mathrm{hr}$. Upon close examination of the HPLC chromatograms, a small amount of the nitrile, $2 \mathbf{a}$, and a peak which co-eluted with the carboxamide (2b) were observed at $t=0$. These trace amounts most likely were impurities in $\mathbf{2 c}$ that were not detected by either ${ }^{1} \mathrm{H}$ NMR or $\mathrm{C}-\mathrm{H}-\mathrm{N}$ analysis used to characterize $2 \mathrm{c}$. It should be noted that the peak $2 \mathrm{~b}$ did not change over the 144-hr incubation period (Fig. 2), indicating that the carboxamide was not an intermediate in the conversion of $\mathbf{2 c}$ to $2 \mathrm{a}$. Further HPLC analysis demonstrated that incubation of the nitrile (2a) and the carboxamide ( $2 \mathbf{b})$ for 4 days under the same conditions as those used for $2 \mathrm{c}$ resulted in no change from the $t=0$ elution pattern. This indicated that 


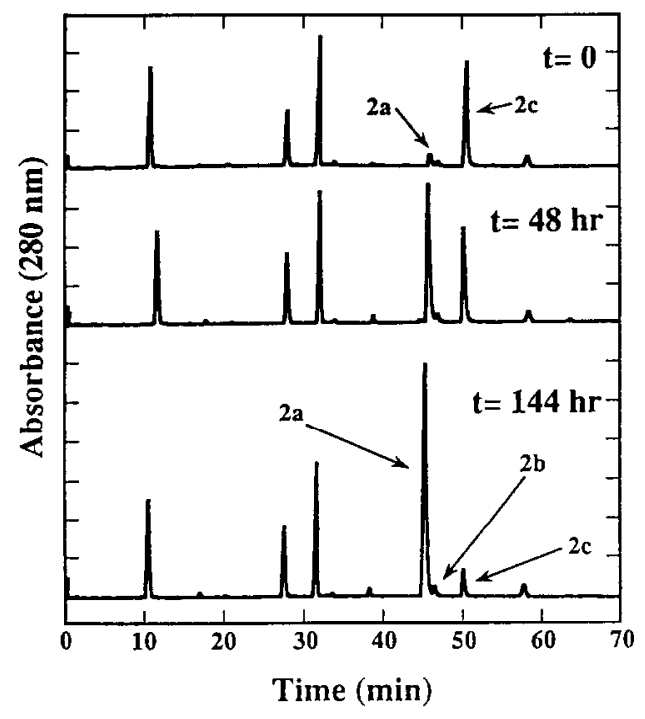

Fig. 2. Chromatograms of Fischer's medium without serum incubated for 0,48 and $144 \mathrm{hr}$ at $37^{\circ}$ with $100 \mu \mathrm{M} \mathrm{2c}$. Absorbance of the effluent was monitored simultaneously at 280 and $294 \mathrm{~nm}$; absorbance at $280 \mathrm{~nm}$ is presented. Peaks other than those labeled $\mathbf{2 a}, \mathbf{2 b}$ or $\mathbf{2} \mathbf{c}$ are from components of Fischer's medium. These peaks were unchanged during the experiment and were observed in a sample not incubated with 2c. Key: (2a) 4-amino-7(benzyl)pyrrolo[2,3- $d$ ]pyrimidine-5-carbonitrile; and (2b and $2 \mathrm{c}$ ) the corresponding 5-carboxamide and 5-thiocarboxamide, respectively.

these two compounds were stable under the culture conditions. These results further demonstrate that the carboxamide cannot be an intermediate in the conversion of the thioamide to the nitrile. Additional studies in which $2 \mathbf{c}$ was incubated in medium containing serum and with cultured $\mathrm{KB}$ cells gave results similar to those obtained for $2 \mathrm{c}$ in cell and serum-free medium.

These results prompted us to examine whether the conversion in cell culture medium of a thioamide moiety to a nitrile was a general phenomenon with 7-substituted 4-aminopyrrolo[2,3- $d]$ pyrimidine-5thioamides. To test this question, we investigated the stability of a related series of compounds, $3 \mathbf{a}-\mathbf{c}$ (Table 1). We chose this series for two reasons: (i) the ether substituent at $\mathrm{N}-7$ of $3 \mathrm{a}-\mathrm{c}$ was structurally different than the benzyl group of $\mathbf{2 a - c}$ and (ii) in contrast to $\mathbf{2 a - c}, \mathbf{3 a - c}$ were non-toxic at $100 \mu \mathrm{M}$ in both KB and L1210 cells (Table 1). Compound 3c was incubated for 5 days under the conditions described previously. HPLC analysis demonstrated that $3 \mathrm{c}$ was converted to a single product that coeluted with the nitrile, 3a, with a $T_{1 / 2}$ of $50 \mathrm{hr}$. In contrast to the studies with $2 \mathbf{c}$, a peak that co-eluted with the carboxamide, $\mathbf{3 b}$, was not detected from $\mathrm{t}=0$ through the 5-day incubation period. As with compound $\mathbf{2 a}$, compound $\mathbf{3 a}$ was stable under the incubation conditions as monitored by HPLC.

Based upon these findings, the stability of the parent nucleosides toyocamycin (1a), sangivamycin

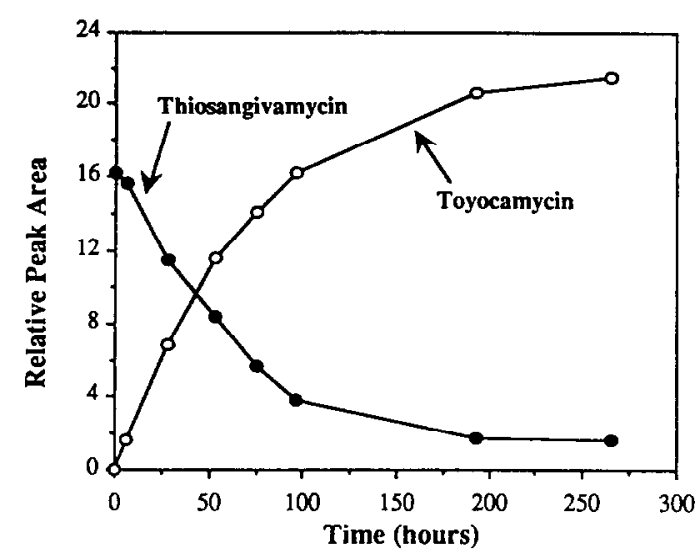

Fig. 3. Stability of the pyrrolopyrimidine nucleoside thiosangivamycin in cell culture medium. Thiosangivamycin was incubated in Fischer's medium without serum for 11 days. Aliquots were removed at the times indicated and analyzed by IIPLC. The peak areas presented in the figure are those determined at the $\lambda_{\max }$ for each nucleoside ( $274 \mathrm{~nm}$ for toyocamycin and $292 \mathrm{~nm}$ for thiosangivamycin). The peak area at later time points for toyocamycin is greater than the peak area for thiosangivamycin at $t=0$ because the molar absorptivity $(\varepsilon)$ for toyocamycin at $274 \mathrm{~nm}$ is greater than the $\varepsilon$ for thiosangivamycin at $292 \mathrm{~nm}$ $[3,4]$.

(1b) and thiosangivamycin (1c) was also studied. Like studies with $\mathbf{2 c}$ and $\mathbf{3 c}$, the stability of thiosangivamycin was examined by incubating it in Fischer's medium without serum for 11 days and analyzing samples by HPLC at selected times during this period. The results showed (Fig. 3) that like $2 \mathrm{c}$ and $3 \mathrm{c}$, thiosangivamycin was converted to a product that co-eluted with toyocamycin (1a) with a $\mathrm{T}_{1 / 2}=$ $55 \mathrm{hr}$. Similar results were obtained by incubating the compound in MEM without serum $\left(T_{1 / 2}=41 \mathrm{hr}\right)$. Like the studies with $3 \mathrm{c}$, a peak that co-eluted with sangivamycin (1b) was not detected from $t=0$ through the 11-day incubation period. As with 2ab and 3a, toyocamycin and sangivamycin were stable under the incubation conditions.

To examine the possible role that components of cell culture medium had on the conversion of the thioamide to the nitrile, a number of studies using buffered solutions or modified cell culture medium were undertaken. The results showed that contrary to those obtained in Fischer's medium and MEM, incubation of 1c in HBS resulted in only a very slow conversion to the nitrile $\left(\mathrm{T}_{1 / 2}>504 \mathrm{hr}\right)$. This conversion of thiosangivamycin (1c) to the corresponding nitrile in HBS was $>10$ times slower than in cell culture medium, strongly suggesting that components contained in culture medium but not in IIBS catalyzed the conversion. To investigate the role in the conversion of certain components in the medium, the $\mathrm{pH}$ of the HBS solution containing Ic was increased from 6.5 to 7.6 , but this did not affect the stability of the compound. Additionally, the benzyl derivative $2 \mathrm{c}$ was incubated with MEM, which did not contain phenol red and glutamine. In this 
Table 3. Structure, cytotoxicity and stability of several 4-substituted pyrrolo[2,3-d]pyrimidine analogs of compound $3 c$

\begin{tabular}{|c|c|c|c|c|c|}
\hline \multirow{2}{*}{ Compound } & \multirow[b]{2}{*}{$\mathrm{R}_{1}$} & \multirow[b]{2}{*}{$\mathrm{R}_{2}$} & \multicolumn{2}{|c|}{ Cytotoxicity, $\mathrm{IC}_{S \mathrm{SO}}(\mu \mathrm{M})$} & \multirow[b]{2}{*}{ Half-life (hr) } \\
\hline & & & $\mathrm{KB}$ & L1210 & \\
\hline $4 a$ & HNMe & $\mathrm{CN}$ & $>100$ & $>100$ & $\mathrm{NA}^{*}$ \\
\hline b & HNMe & $\mathrm{CSNH}_{2}$ & $>100$ & $>100$ & 20 \\
\hline $5 \mathbf{a}$ & $\mathrm{N}(\mathrm{Me})_{2}$ & $\mathrm{CN}$ & $>100$ & $>100$ & NA \\
\hline b & $\mathrm{N}(\mathrm{Me})_{2}$ & $\mathrm{CSNH}_{2}$ & $>100$ & $>100$ & 150 \\
\hline $6 a$ & $\mathrm{H}$ & $\mathrm{CN}$ & $>100$ & $>100$ & NA \\
\hline b & $\mathbf{H}$ & $\mathrm{CSNH}_{2}$ & $>100$ & $>100$ & 175 \\
\hline
\end{tabular}

Cytotoxicity was determined by measuring the effects of compounds on the growth of KB and L1210 cells. Data are the averages of two or more experiments. The greater than symbol indicates that the $\mathrm{IC}_{50}$ was not reached at the highest concentration tested.

* Not applicable.

medium, the $T_{1 / 2}$ was extended from $41 \mathrm{hr}$ to approximately $65 \mathrm{hr}$ demonstrating that although these two components affected the stability of the thioamide moiety, they could not completely account for the conversion.

To investigate whether changes on the heterocycle at sites other than $\mathrm{N}-7$ could affect the stability of the thioamide group, we examined the effect of modifications at the 4-position of $3 c$ on the half-life. In this study, the 4-amino group of compound 3 was replaced with a proton $(\mathrm{H})$, a methylamino group $\left(\mathrm{NHCH}_{3}\right)$ or a dimethylamino group $\left(\mathrm{N}\left(\mathrm{CII}_{3}\right)_{2}\right)-$ compounds $4-6$ (Table 3 ). Like compounds $3 a-c$, compounds 4-6 were essentially non-toxic in both $\mathrm{KB}$ and $\mathrm{L} 1210$ cells with $\mathrm{IC}_{50}$ values $>100 \mu \mathrm{M}$ (Table 3). Like compounds 1-3, compounds 4-6 were incubated in Fischer's medium, and their stability was monitored by HPLC. The results demonstrated that modifications at the 4-position have a definite effect on the stability of the 5-thioamide moiety, whereas changes at N-7 tend not to affect the stability of the 5-thioamide moiety (Table 3). For example, replacement of the 4-amino group of $3 c$ with a methylamino group (4b) decreased the stability of the thioamide $\left(T_{1 / 2}=20 \mathrm{hr}\right)$. In contrast, replacement with a dimethylamino group (5b) or a hydrogen (6b) extended the $T_{1 / 2}$ from $50 \mathrm{hr}$ for $3 \mathrm{c}$ to $150 \mathrm{hr}$ for $\mathbf{5 b}$ and $175 \mathrm{hr}$ for $\mathbf{6 b}$. In the case of $\mathbf{5 b}$, we also observed an unidentified peak by HPLC in addition to $5 \mathbf{a}$ and $\mathbf{5 b}$. This possible intermediate appeared $24 \mathrm{hr}$ into the incubation and rapidly decreased thereafter. This was the only compound during this entire study where we observed an HPLC peak other than the thioamide or nitrile analogs.

\section{DISCUSSION}

The present study has established that the pyrrolopyrimidine nucleoside thiosangivamycin and several related analogs are converted to the corresponding nitrile analogs in cell culture medium. The conversion of thiosangivamycin to toyocamycin in cell culture medium has not, to the best of our knowledge, been observed previously. Even if the conversion had occurred in previous studies, there would have been no obvious reason to suspect this since thiosangivamycin and toyocamycin are equally toxic to mammalian cells. The toxicity of thiosangivamycin and toyocamycin arises because both compounds are phosphorylated by adenosine kinase [24] to afford the 5 '-monophosphate derivatives that ultimately are incorporated into DNA or RNA $[5,6]$. Whether the 5'-monophosphate of thiosangivamycin is converted to phosphorylated toyocamycin is unknown and was not included in the present study. However, our results, vide supra, suggest that this would occur since a variation of the substituent at N-7 does not seem to have a major effect on the conversion.

It is interesting that changes at the 4-position can have a major effect on the stability of the 5thiocarboxamide moiety. This would suggest that the 4-position may play a key role in stabilizing or destabilizing the 5-thioamide group. Results obtained for the 4-substituted amino derivatives ( $4 \mathbf{b}$ and $\mathbf{5 b}$ ) revealed that increasing the basicity of the amino group at C-4 does not necessarily correlate with an increase or decrease in the stability of the thioamide. Instead, the stability of a substituted thioamide compound seems directly related to the presence or absence of an amino group with a free proton at C-4. Whether modifications at other sites on the heterocycle could affect the stability of the thioamide group is unknown.

The rapid conversion of $\mathbf{2 c}$ to its corresponding 
nitrile (2a) in cell culture medium raises an interesting issue regarding the antiviral activity. We have demonstrated [23] that 2a is inactive against HCMV at concentrations up to $100 \mu \mathrm{M}$, whereas $2 \mathrm{c}$ has potent activity against $\mathrm{HCMV}$ with an $\mathrm{IC}_{50}=0.4 \mu \mathrm{M}$. Our present results suggest that the action of $\mathbf{2 c}$ against HCMV must occur rather rapidly since by $48 \mathrm{hr}$ essentially $50 \%$ of the active compound is converted to an inactive one. We provided evidence for this hypothesis while studying the temporal effect of $2 c$ on DNA and RNA synthesis [23]. We showed that in uninfected cells, various concentrations of $2 \mathrm{c}$ dramatically affect both DNA and RNA syntheses approximately $1-2 \mathrm{hr}$ following its addition to cultured CEM-SS cells. These results, however, were obtained in uninfected cells and whether the potent early inhibition of RNA and DNA syntheses by 2c in uninfected cells correlates with the potent activity against HCMV has yet to be established and is currently under investigation.

Beside the nitrilc derivatives, the identity of any other product involved in the conversion is unknown. Since 1c, 2c, 3c and $\mathbf{4 b}$ exhibit similar stability patterns but completely different cytotoxicity profiles, it is highly unlikely that the release of $\mathrm{H}_{2} \mathrm{~S}$ as a byproduct of the conversion caused cell toxicity. Regarding the causes of this phenomenon, the identification of a single component has proved to be difficult. Our results suggest that there may be a combination of components, such as phenol red and glutamine, that are involved in catalyzing the observed conversion. Chemically, thioamides can be converted to nitriles by treatment with mercuric chloride and methylamine $[25,26]$. In the current study, these reagents were not present during the incubation of any of the thioamide-substituted derivatives. There have been reports, however, of more mild reaction conditions furnishing a nitrile from a thioamide $[4,27]$. Specifically, alkylation of thiosangivamycin (1c) with methyl iodide afforded a 5-methylthioformimidate intermediate that rapidly eliminated methanethiol to give toyocamycin (1a). Similar results were obtained upon treatment of 5 amino - 4 - thiocarboxamide - $1-\beta$ - D - ribofuranosylimidazole (thio-AICA-riboside) with methyl iodide. From this information it may not be entirely unreasonable to propose the formation of an alkylated thio-intermediate that can rapidly eliminate to give the nitrile. The possible appearance of an intermediate in the conversion of 4-dimethylamino7 - [(2-methoxyethoxy)methyl]pyrrolo[2,3-d]pyrimidine-5-thiocarboxamide (5b) to the corresponding nitrile (5a) may support this argument; however, the exact mechanism of how the thioamide is converted to the nitrile in cell culture medium is unknown.

In summary, our results have demonstrated that the 5-thioamide moiety of many 7-substituted 4aminopyrrolo $[2,3-d]$ pyrimidines is unstable in cell culture medium, and is converted to the corresponding 5-nitrile regardless of the substituent at $\mathrm{N}-7$. In contrast, substituents at the 4-position of the heterocycle significantly affect the stability of the 5thioamide moiety. Investigators using these or structurally similar compounds should be aware of these results and appreciate that biological data generated in cell culture may be due to a mixture of the thioamide and by-product(s) of the conversion.

Acknowledgements-We thank Julie Breitenbach and Patricia Whittler for their expert technical assistance. This research was supported by Department of Health and Human Services Research Contract N01-A172641 and grant U01-AI 31718 and American Cancer Society Research Grant DHP-36.

\section{REFERENCES}

1. Nishimura $H$, Katagiri $K$, Sato $K$, Mayama $M$ and Shimaoka $N$, Toyocamycin, a new anti-candidia antibiotic. J Antibiot (Tokyo) 9A: 60-62, 1956.

2. Rao KV and Renn DW, BA-90912: An antitumor substance. Antimicrob Agents Chemother 77-79, 1963.

3. Tolman RL, Robins RK and Townsend LB, Pyrrolopyrimidine nucleosides. III. The total synthcsis of toyocamycin, sangivamycin, tubercidin and related derivatives. I Am Chem Soc 91: 2102-2108, 1969

4. Hinshaw BC, Gerster JF, Robins RK and Townsend LB, Pyrrolopyrimidine nucleosides. V. A study on the relative chemical reactivity of the 5-cyano group of the nucleoside antibiotic toyocamycin and desaminotoyocamycin. The synthesis of analogs of sangivamycin. J Org Chem 35: 236-241, 1970.

5. Glazer RI and Peale AL, Comparison between the inhibitory activities of sangivamycin and thiosangivamycin on nuclear ribonucleic acid synthesis in L1210 cells in vitro. Biochem Pharmacol 29: 305-310, 1980.

6. Ritch PS and Helmsworth M, Pyrrolopyrimidine lethality in relation to ribonucleic acid synthesis in Sarcoma 180 cells in vitro. Biochem Pharmacol 31: 2686-2688, 1982.

7. Cavins JA, Hall TC, Olson KB, Khung CL. Horton J, Colsky $J$ and Shadduck RK, Initial toxicity study of sangivamycin (NSC-65346). Cancer Chemother Rep 51: 197-200, 1967

8. Turk SR, Shipman C Jr, Nassiri MR, Genzlinger G, Krawczyk SH, Townsend LB and Drach JC, Pyrrolo[2,3-d]pyrimidine nucleosides as inhibitors of human cytomegalovirus. Antimicrob Agents Chemother 31: $544-550,1987$.

9. Gupta PK, Daunert S, Nassiri MR, Wotring LL, Drach $\mathrm{JC}$ and Townsend LB, Synthesis, cytotoxicity and antiviral activity of some acyclic analogues of the pyrrolo[2,3-d]pyrimidine nucleoside antibiotics tubercidin, toyocamycin and sangivamycin. $J \mathrm{Med}$ Chem 32: 402-408, 1989.

10. Gupta PK, Nassiri MR, Coleman LA, Wotring LL, Drach JC and Townsend LB, Synthesis, cytotoxicity. and antiviral activity of certain 7-[(2-hydroxyethoxy)methyl]pyrrolo[2,3-d]pyrimidine nucleosides related to toyocamycin and sangivamycin. J Med Chem 32: 1420-1425, 1989.

11. Birch GM, Krawczyk SH, Townsend LB and Drach JC, Antagonism of the cytotoxic but not antiviral effects of ara-sangivamycin by adenosine. Antimicrob Agents Chemother 33: 1606-1608, 1989.

12. Saxena NK, Coleman I.A. Drach IC and Townsend LB, Synthesis and antiviral activity of some 7(2 - hydroxyethoxymethyl)pyrazolo $[3,4-d]$ pyrimidine analogs of sangivamycin and toyocamycin. $J$ Med Chem 33: $1980-1983,1990$.

13. Townsend LB, Drach JC. Wotring LL, Vittori S, Pudlo JS, Swayze EE, Gupta P, Maruyama T, Saxena N, Coleman L, Westerman AC, Spurr J, Nassiri MR, Turk SR and Krawczyk SH, Design, synthesis, and studies on the structure activity relationships of certain pyrrolo $[2,3-d \mid$ pyrimidine nucleosides and structurally 
related analogs as potential antineoplastic and antiviral agents. Farmaco 46: 113-139, 1991.

14. Renau TE, Nassiri MR, Swayze EE, Townsend LB and Drach JC, Improved synthesis and biological evaluation of an acyclic thiosangivamycin active against human cytomegalovirus. Antiviral Res 19: 15-28, 1992.

15. Renau TE, Ludwig MS, Drach JC and Townsend LB, Design, synthesis and activity against human cytomegalovirus of non-phosphorylatable analogs of toyocamycin, sangivamycin and thiosangivamycin. Biorg Med Chem Lett 2: 1755-1760, 1992.

16. Swayze EE, Hinkley JM and Townsend LB, 2Amino-5-bromo-3, 4-dicyanopyrrole. In: Nucleic Acid Chemistry; Improved and New Synthetic Procedures, Methods and Techniques, Part IV (Eds. Townsend LB and Tipson RS), pp. 16-18. Wiley-Interscience, New York, 1991.

17. Hinshaw BC, Leonoudakis O, Schram KH and Townsend LB, Pyrrolopyrimidine nucleosides. Part X. Synthesis of certain 4,5-disubstituted 7-( $\beta-\mathrm{D}$ ribofuranosyl)-pyrrolo[2,3-d]pyrimidines related to toyocamycin and sangivamycin. J Chem Soc Perkin Trans I 1248-1253, 1975.

18. Prichard MN, Prichard LE, Baguley WA, Nassiri MR and Shipman $\mathrm{C} J \mathbf{r}$, Three-dimensional analysis of the synergistic cytotoxicity of ganciclovir and zidovudine. Antimicrob Agents Chemother 35: 1060-1065, 1991.

19. Wotring LL and Townsend LB, Study of the cytotoxicity and metabolism of 4-amino-3-carboxamido-1- $(\beta$ D - ribofuranosyl)pyrazolo[3,4 - $d]$ pyrimidine using inhibitors of adenosine kinase and adenosine deaminase. Cancer Res 39: 3018-3023, 1979.

20. Nassiri MR, Hudson JL, Pudlo JS, Birch GM,
Townsend LB and Drach JC, Flow cytometric evaluation of the cytotoxicity of novel antiviral compounds. Cytometry 11: 411-417, 1990.

21. Shipman C Jr, Control of culture $\mathrm{pH}$ with synthetic buffers. In: Tissue Culture. Methods and Applications (Eds. Kruse PF Jr and Patterson MK Jr), pp. 709-712. Academic Press, New York, 1973.

22. Krstulović AM and Brown PR, Reversed-Phase High Performance Liquid Chromatography, pp. 236-241. Wiley-Interscience, New York, 1982.

23. Renau TE, Young CG, Nassiri MR, Lee JS, Wotring LL, Townsend LB and Drach JC, Antiherpetic activity, cytotoxicity and metabolism of non-nucleoside analogs related to toyocamycin, sangivamycin and thiosangivamycin. Antiviral Res [Suppl] 1: 118, 1993.

24. Miller RL, Adamczyk DL, Miller WH, Koszalka GW, Rideout JL, Beacham LM, Chao EY, Haggerty JJ, Krenitsky TA and Elion GB, Adenosine kinase from rabbit liver. II. Substrate and inhibitor specificity. $J$ Biol Chem 254: 2346-2352, 1979.

25. Shaw G and Butler DN, Purines, pyrimidines and glyoxalines. Part XIV. The preparation and some reactions of $\alpha$-amino- $\alpha$-cyanothioacetamide, leading to 6-mercaptopurines and adenines. J Chem Soc 4040$4045,1959$.

26. Earl RA and Townsend LB, Pyrazolopyrimidine nucleosides. Part VII (1). The synthesis of certain pyrazolo[3,4- $d]$ pyrimidine nucleosides related to the nucleoside antibiotics toyocamycin and sangivamycin. J Heterocycl Chem 11: 1033-1039, 1974.

27. Yamazaki A, Kumashiro I, Takenishi T and Ikehara $\mathrm{M}$, Synthesis of thio-aica, 6-thioxanthine, isoguanine analogs and their ribosides. Chem Pharm Bull (Tokyo) 16: 2172-2181, 1968. 